\title{
THE TRANSFORMS OF FUCHSIAN GROUPS
}

\author{
P. K. REES
}

This paper will deal with Fuchsian linear transformations that have the unit circle

$$
Q_{0}(z) \equiv z \bar{z}-1=0
$$

as principal circle. The transformations have the form

$$
T(z)=\frac{a z+\bar{c}}{c z+\bar{a}}, \quad a \bar{a}-c \bar{c}=1 .
$$

We shall develop several theorems concerning the relative size of the isometric circle of any transformation $T$ of the group and of the isometric circle of

$$
\begin{aligned}
S(z) & =G T G^{-1}(z) \\
& =\frac{(-\alpha \bar{\alpha} a+\alpha \nu \bar{c}-\bar{\alpha} \bar{\nu} c+\nu \bar{\nu} \bar{a}) z+\alpha \bar{\nu} a-\alpha^{2} \bar{c}+\bar{\nu}^{2} c-\alpha \bar{\nu} \bar{a}}{\left(-\bar{\alpha} \nu a+\nu^{2} \bar{c}-\bar{\alpha}^{2} c+\bar{\alpha} \nu \bar{a}\right) z+\nu \bar{\nu} a-\alpha \nu \bar{c}+\bar{\alpha} \bar{\nu} c-\alpha \bar{\alpha} \bar{a}} \\
& =\frac{A z+\bar{C}}{C z+\bar{A}}, \quad A \bar{A}-C \bar{C}=1,
\end{aligned}
$$

in which

is variable and $T$ fixed.

$$
G(z)=\frac{\alpha z+\bar{\nu}}{\nu z+\bar{\alpha}}, \quad \alpha \bar{\alpha}-\nu \bar{\nu}=1,
$$

The concept of the isometric circle of a linear transformation was introduced in 1926 by L. R. Ford. Because of his work with it, the isometric circle now plays a fundamental part in work with groups of linear transformations. By definition* the isometric circle of a linear transformation is the complete locus of points in the neighborhood of which lengths and areas are unaltered in magnitude by the transformation. For convenience we shall sometimes use the designation $Q(z)$ for a line or circle $Q(z)=0$.

THEOREM 1. A necessary and sufficient condition that the radii $r_{s}$ and $r_{t}$ of the isometric circles of $S$ and $T$ be equal is that the center w of the isometric circle of the transformation $G$ be either on the line $Q_{1}(z)$ through the center $g_{t}$ of the isometric circle of $T$ and the center $g_{t}^{\prime}$ of the isometric circle of $T^{-1}$ or on the inverse $Q_{2}(z)$ of $Q_{1}(z)$ in $Q_{0}(z)$.

* L. R. Ford, Automorphic Functions, p. 25. 
Proof. The equation of $Q_{1}$ is

$$
Q_{1}(z)=c z-\bar{c} \bar{z}-(a-\bar{a})=0 .
$$

The equation of $Q_{2}$, which is the inverse of $Q_{1}$ in $Q_{0}$, is

$$
Q_{2}(z)=c z-\bar{c} \bar{z}-(a-\bar{a}) z \bar{z}=0 .
$$

If $z$ lies on either $Q_{1}$ or $Q_{2}$, we have

$$
\begin{aligned}
Q_{1}(z) Q_{2}(z)= & -(a-\bar{a})(c z-\bar{c} \bar{z})(z \bar{z}+1)+z \bar{z}\left[(a-\bar{a})^{2}-2 c \bar{c}\right] \\
& +c^{2} z^{2}+\bar{c}^{2} \bar{z}^{2}=0 .
\end{aligned}
$$

Now, using the values of $r_{t}$ and $r_{s}$ from (1) and (2), we have

$$
\begin{aligned}
\frac{1}{r_{s}^{2}}-\frac{1}{r_{t}^{2}}= & C \bar{C}-c \bar{c}=-(a-\bar{a})(-\alpha \nu \bar{c}+\bar{\alpha} \bar{\nu} c)(\alpha \bar{\alpha}+\nu \bar{\nu}) \\
& -\alpha \bar{\alpha} \nu \bar{\nu}(a-\bar{a})^{2}-\alpha^{2} \nu^{2} \bar{c}^{2}-\bar{\alpha}^{2} \bar{\nu}^{2} c^{2}+\left(\alpha^{2} \bar{\alpha}^{2}+\nu^{2} \bar{\nu}^{2}-1\right) c \bar{c} \\
= & -(a-\bar{a})(-\alpha \nu \bar{c}+\overline{\bar{\nu}} \bar{\nu} c)(\alpha \bar{\alpha}+\nu \bar{\nu}) \\
& -\alpha \bar{\alpha} \nu \bar{\nu}\left[(a-\bar{a})^{2}-2 c \bar{c}\right]-\alpha^{2} \nu^{2} \bar{c}^{2}-\bar{\alpha}^{2} \bar{\nu}^{2} c^{2}
\end{aligned}
$$

which vanishes if and only if $r_{s}=r_{t}$.

Dividing (5) equated to zero by $-(\nu \bar{\nu})^{2}$, replacing $-\bar{\alpha} / \nu$ by $w$, and comparing the result with (3), we see that $r_{s}=r_{t}$ if and only if $w$ lies on $Q_{1}(z)$ or $Q_{2}(z)$.

THEOREM 2a. A necessary and sufficient condition that $r_{s}<r_{t}$ $\left(r_{s}>r_{t}\right)$ is that $z=w$, substituted in the expression for $Q_{1} Q_{2}$, make that expression negative (positive).

PROOF. $r_{s}<r_{t}\left(r_{s}>r_{t}\right)$ according as $1 / r_{s}^{2}-1 / r_{t}{ }^{2}>0\left(1 / r_{s}^{2}-1 / r_{t}^{2}<0\right)$. However,

$$
Q_{1} Q_{2}=\left(1 / r_{s}^{2}-1 / r_{t}^{2}\right) /(-\nu \bar{\nu})^{2} .
$$

Therefore, $Q_{1} Q_{2}<0$ or $Q_{1} Q_{2}>0$ according as $1 / r_{s}{ }^{2}-1 / r_{t}{ }^{2}>0$ or $1 / r_{s}^{2}-1 / r_{t}^{2}<0$.

THEOREM 2b. A necessary and sufficient condition that $r_{s}>r_{t}$ is that $w$ be outside $Q_{2}$ and on the opposite side of $Q_{1}$ from the origin; a necessary and sufficient condition that $r_{s}<r_{t}$ is that $w$ be inside $Q_{2}$ and on the opposite side of $Q_{1}$ from the origin or outside $Q_{2}$ and on the same side of $Q_{1}$ as the origin.*

Proof. The function $Q_{1}$ is positive (negative) according as $w$ is on the opposite (same) side of $Q_{1}$ from (as) the origin. The function $Q_{2}$

* The case of $w$ inside $Q_{2}$ and on the same side of $Q_{1}$ as the origin is omitted since then $w$ would be inside $Q_{0}$. 
is positive (negative) according as $w$ is outside (inside) $Q_{2}$. Comparing these statements we have the theorem:

THEOREM 3. A necessary and sufficient condition that $r_{s}=r_{t} / k$, $(k \geqq 0)$, is that w lie on the locus of the equation

$$
\begin{aligned}
k^{2}(z \bar{z}-1)^{2} & =\left[z^{2}-(a-\bar{a}) \frac{z}{c}-\frac{\bar{c}}{c}\right]\left[\bar{z}^{2}+(a-\bar{a}) \frac{\bar{z}}{\bar{c}}-\frac{c}{\bar{c}}\right] \\
& =\left|z-\xi_{1}\right|^{2}\left|z-\xi_{2}\right|^{2},
\end{aligned}
$$

where $\xi_{1}$ and $\xi_{2}$ are the fixed points of the transformation $T$.

Proof. From (4), by adding $1 / r_{t}{ }^{2}$ to each member, multiplying by $r_{t}^{2} /(\nu \bar{\nu})^{2}$, putting $w=-\bar{\alpha} / \nu$, making use of the relation $\alpha \bar{\alpha}-\nu \bar{\nu}=1$, putting $r_{t} / r_{s}=k$, and factoring the right-hand member, we get

$$
k^{2}(w \bar{w}-1)^{2}=\left|w-\xi_{1}\right|^{2}\left|w-\xi_{2}\right|^{2},
$$

where

$$
\xi_{1}, \xi_{2}=\left\{(a-\bar{a}) \pm\left[(a-\bar{a})^{2}+4 c \bar{c}\right]^{1 / 2}\right\} / 2 c .
$$

THEOREM 4. If $T$ is a hyperbolic transformation, the minimum value of $k$ for all transformations $G$ is given by

$$
k_{0}^{2}=\frac{1}{4 c \bar{c}}\left[(a-\bar{a})^{2}+4 c \bar{c}\right]=\frac{1}{4}\left|\xi_{1}-\xi_{2}\right|^{2} ;
$$

this value of $k$ is taken on if and only if $w$ is on the circle

$$
Q_{3,4}(z) \equiv \frac{1}{2}(a-\bar{a}) i z \bar{z}-i c z+i \bar{c} \bar{z}+(a-\bar{a}) \frac{i}{2} ;
$$

for parabolic transformations zero is the lower bound for $k$; for elliptic transformations the minimum value of $k$ is zero, and this value is taken on if and only if $w=\xi_{1}$.

Proof. Let us consider

$$
k_{0}^{2}=\frac{1}{4 c \bar{c}}\left[(a-\bar{a})^{2}+4 c \bar{c}\right] .
$$

From (6) with the right-hand member expanded in terms of the coefficients of $T$, we have

$$
\begin{aligned}
c \bar{c}(w \bar{w}- & 1)^{2}\left(k^{2}-k_{0}{ }^{2}\right) \\
= & (a-\bar{a})(c w-\bar{c} \bar{w})(w \bar{w}+1)-w \bar{w}(a-\bar{a})^{2}-c^{2} w^{2}-\bar{c}^{2} \bar{w}^{2} \\
& +\left(w^{2} \bar{w}^{2}+1\right) c \bar{c}-(w \bar{w}-1)^{2}(a-\bar{a})^{2} / 4-(w \bar{w}-1)^{2} c \bar{c}
\end{aligned}
$$




$$
\begin{aligned}
& =\left[\frac{1}{2}(a-\bar{a}) i w \bar{w}-i c w+i \bar{c} \bar{w}+(a-\bar{a}) \frac{i}{2}\right]^{2} \\
& =\left[Q_{3,4}(w)\right]^{2} \geqq 0
\end{aligned}
$$

for all $w$, since the expression in brackets is real. Hence, $k^{2}=k_{0}{ }^{2}$ if and only if $w$ is on $Q_{3,4}$ since $|w| \neq 1$ and $c \neq 0$.

Let us notice that $k^{2}$ cannot have the value $k_{0}^{2}$ for elliptic transformations, since in that case $(a-\bar{a})^{2}+4 c c<0$ whereas $k$ is a non-negative real number. Furthermore, $Q_{3,4}(w)$ has no real locus for $T$ an elliptic transformation.

From the explicit expression for $Q_{3,4}(w)$ in the non-elliptic case it is obviously a circle with center at $-2 \bar{c} /(a-\bar{a})$, $a$ not real, and radius equal to $\left\{ \pm i\left[(a-\bar{a})^{2}+4 c \bar{c}\right]^{1 / 2}\right\} /(a-\bar{a})$, the sign being so chosen that the radius is positive.

Let us notice that $r_{3,4}=0$ for $T$ parabolic, also that $Q_{3,4}(\xi)=0$, where $\xi=(a-\bar{a}) / 2 c$ is the fixed point of the transformation. Now for parabolic transformations we have $Q_{0}(\xi)=0$; hence $\xi$ is not a permissible value for $w$.

From (6) it is obvious that $k=0$ for elliptic transformations and that $w=\xi_{1}, \xi_{2}$. However, $w \neq \xi_{2}$ since $Q_{0}\left(\xi_{2}\right)<0$. We merely call attention to the fact that $S$ is a rotation when $w$ is equal to $\xi_{1}$, the fixed point of $T$ which is outside $Q_{0}$.

For hyperbolic transformations $\xi_{1}$ and $\xi_{2}$ are on $Q_{0}$ and hence are not permissable values of $w$.

Let us now consider $k_{0}$ when $a$ is real. From the expression given in the theorem for $k_{0}$ we see that it is unity for $a$ real. From (6) it follows immediately that

$$
\left(k^{2}-1\right)(w \bar{w}-1)^{2} c \bar{c}=-(c w-\bar{c} \bar{w})^{2} \geqq 0
$$

for all $w$ since $c w-\bar{c} \bar{w}$ is a pure imaginary. Hence, for $a$ real the minimum value of $k$ is unity and is taken on if and only if $w$ is on $Q_{3,4} \equiv Q_{1} \equiv Q_{2}$. We call attention to the fact that if $a$ is real, the transformation is hyperbolic since $g_{t}$ and $g_{t}^{\prime}$ are on opposite ends of a diameter of $Q_{0}$.

It is readily seen from (6), with $w$ and $\bar{w}$ as independent variables, that there are no relative maximum and minimum values of $k$.

REMARK. If $G$ is so chosen that $k=k_{0}$, then no transform of $S$ will increase the magnitude of its isometric circle. This is apparent since applying $G_{2}$ to $S=G_{1} T G_{1}^{-1}$ is equivalent to applying $G_{3}=G_{2} G_{1}$ to $T$, but $G_{1}$ is such that $I_{t}$ receives its maximum magnification.

COROLlaRY $Q_{3,4}$ is self-inverse with respect to $Q_{0}$. 
Proof. Replacing $z$ and $\bar{z}$ in $Q_{3,4}$ by $1 / \bar{z}$ and $1 / z$, respectively, and multiplying by $z \bar{z}$, we get the equation $Q_{3,4}(z)=0$.

We found in Theorem 3 a necessary and sufficient condition that $r_{s}=r_{t} / k$ to be that $w$ satisfy the equation.

$$
k^{2}(z \bar{z}-1)^{2}=\left|z-\xi_{1}\right|^{2}\left|z-\xi_{2}\right|^{2} .
$$

We shall now investigate the geometric significance of that equation. Let

$$
\begin{aligned}
& M=(a-\bar{a})^{2}+4 c \bar{c} \\
& B_{3}=-\left[(a-\bar{a})+\left(M-4 k^{2} c \bar{c}\right)^{1 / 2}\right] / 2 \bar{c}\left(k^{2}-1\right), \\
& B_{4}=-\left[(a-\bar{a})-\left(M-4 k^{2} c \bar{c}\right)^{1 / 2}\right] / 2 \bar{c}\left(k^{2}-1\right), \\
& C_{3}=B_{3} / B_{4}=\left[a-\bar{a}+\left(M-4 k^{2} c \bar{c}\right)^{1 / 2}\right]^{2} / 4 c \bar{c}\left(k^{2}-1\right), \\
& C_{4}=B_{4} / B_{3}=\left[a-\bar{a}-\left(M-4 k^{2} c \bar{c}\right)^{1 / 2}\right]^{2} / 4 c \bar{c}\left(k^{2}-1\right) .
\end{aligned}
$$

THEOREM 5. A necessary and sufficient condition that $r_{s}=r_{t} / k$, $(k \neq 1)$, is that w lie on either

$$
Q_{3}(z)=z \bar{z}+B_{3} z+\bar{B}_{3} \bar{z}+C_{3}=0
$$

or

$$
Q_{4}(z)=z \bar{z}+B_{4} z+\bar{B}_{4} \bar{z}+C_{4}=0 .
$$

Proof. Multiplying $Q_{3}(z)$ by $Q_{4}(z)$, slightly changing the form of (6), and equating coefficients of like terms, we have the following equations that must be satisfied simultaneously:

(14) $C_{3}+C_{4}+B_{3} \bar{B}_{4}+\bar{B}_{3} B_{4}=\left[(a-\bar{a})^{2}-2 k^{2} c \bar{c}\right] / c \bar{c}\left(k^{2}-1\right)$.

Solving (10), (11), (12), and (13) we get the values stated for $B_{3}$, $B_{4}, C_{3}$, and $C_{4}$ and also $C_{3}=C_{4}=1$. It is readily seen that the given values of $B_{3}, B_{4}$, and $C_{3}=C_{4}=1$ do not satisfy (14). However, the given values of the four symbols do satisfy the five equations.

COROLlary 1. The circles $Q_{3}$ and $Q_{4}$ are always real circles for $T$ an elliptic or a parabolic transformation; they are real circles for $T$ a hyperbolic transformation and $k \geqq k_{0}$, the minimum value of $k$ for $T$ hyperbolic. 
Proof. Since $C_{3}$ and $C_{4}$ are real it is sufficient to show that the radius is real for each of the cases mentioned. The expression under the radical in $B_{3}, B_{4}, C_{3}$, and $C_{4}$ is readily seen to be non-positive for $T$ an elliptic or a parabolic transformation and also for $T$ hyperbolic provided $k \geqq k_{0}$. Therefore,

$$
\begin{aligned}
r_{j}^{2} & =B_{j} \bar{B}_{j}-C_{j}, \quad j=3,4, \\
& =-\frac{\left[a-\bar{a}-\left(M-4 k^{2} c \bar{c}\right)^{1 / 2}\right]^{2} k^{2}}{4 c \bar{c}\left(k^{2}-1\right)^{2}} \geqq 0 .
\end{aligned}
$$

Corollary 2. $Q_{3}$ and $Q_{4}$ are inverse circles with respect to $Q_{0}$.

PROOF. In order to find the inverse of a circle with respect to $Q_{0}$, we need only replace $z$ and $\bar{z}$ by $1 / \bar{z}$ and $1 / z$, respectively, and multiply by $z \bar{z}$. The corollary is immediate upon performing this operation since $C_{3} C_{4}=1, B_{3}=B_{4} / C_{4}$, and $C_{3}$ and $C_{4}$ are real.

CoRollary 3. In the hyperbolic case, the circles $Q_{3}$ and $Q_{4}$ coincide with

$$
Q_{3,4}(z)=\frac{1}{2}(a-\bar{a}) i z \bar{z}-i c z+i \bar{c} \bar{z}+(a-\bar{a}) \frac{i}{2}=0,
$$

when $k$ is equal to $k_{0}$, the minimum value of $k$.

Proof. The expression under the radical in $B_{3}, B_{4}, C_{3}$, and $C_{4}$, when evaluated for $k=k_{0}$, is zero; hence we have $B_{3}=B_{4}$ and $C_{3}=C_{4}=1$ and therefore $Q_{3}=Q_{4}$. Evaluating $B_{3}$ for $k=k_{0}$ and putting this into the equation of $Q_{3}$, along with $C_{3}=1$, we have

$$
Q_{3}(z)=Q_{4}(z)=Q_{3,4}(z) .
$$

COROllary 4. The radius $r_{3}$ of $Q_{3}$ is equal to the product of $k$, the reciprocal of the magnification of $r_{t}$, and the absolute value of the center $-\bar{B}_{3}$ of $Q_{3}$. A similar statement is true for $Q_{4}$.

Proof. Symbolically, the above statement is

$$
r_{3}^{2}=k^{2} B_{3} \bar{B}_{3} .
$$

The validity of this relation is immediate upon recalling that

$$
r_{3}^{2}=B_{3} \bar{B}_{3}-C_{3},
$$

equating the right-hand members of these two equations, and replacing $B_{3}$ and $C_{3}$ by their values as given immediately preceding theorem 5 .

Theorem 6. $Q_{1}, Q_{2}, Q_{3}$, and $Q_{4}$ are fixed circles of the transformation $T$. 
Proof. A necessary and sufficient condition that a given circle be a fixed circle of $T$ is that it be orthogonal to both $I_{l}$ and $I_{t}^{\prime}$.* $^{*}$

Using the law of cosines, we find the angle $\theta$ between any two circles

$$
q_{j}=z \bar{z}+b_{j} z+\bar{b}_{j} \bar{z}+c_{j}=0, \quad j=1,2,
$$

to be given by

$$
\begin{array}{rlr}
\cos \theta & =\frac{1}{2 r_{1} r_{2}}\left[c_{1}+c_{2}-b_{1} \bar{b}_{2}-\bar{b}_{1} b_{2}\right], & \\
r_{j}{ }^{2} & =b_{j} \bar{b}_{j}-c_{j}, & j=1,2 .
\end{array}
$$

Now using the analytic expressions for $Q_{3}$ and $I_{t}$ and substituting in (15), we have $\cos \theta=0$. Hence $Q_{3}$ and $I_{t}$ are orthogonal. Similarly, we find that $Q_{3}$ and $I_{i}^{\prime}$ are orthogonal. Therefore, the circles $Q_{3}$ are fixed circles of the transformation $T$. It can be shown in a similar manner that $Q_{1}, Q_{2}$, and $Q_{4}$ are also fixed circles of $T$.

Theorem 7. For T hyperbolic

$$
k^{2}=\frac{(a-\bar{a})^{2}+4 c \bar{c}}{4 c \bar{c} \sin ^{2} \theta},
$$

where $\theta$ is the angle between $Q_{0}$ and $Q_{4}$.

Proof. We shall use (15), letting $q_{1}(z)=Q_{0}(z)$ and $q_{2}(z)=Q_{4}(z)$. Then

$$
\begin{aligned}
\cos \theta & =\frac{1}{2 r_{4}}\left(-1+C_{4}\right) \\
& =-\frac{\left[1+\left(k^{2}-1\right) B_{4} \bar{B}_{4}\right]}{2 k\left(B_{4} \bar{B}_{4}\right)^{1 / 2}}
\end{aligned}
$$

by Corollary 4 of Theorem 5 . After putting in the value of $B_{4}$ and simplifying, we have

$$
\cos \theta=-\frac{\left[(a-\bar{a})^{2}-4 c \bar{c}\left(k^{2}-1\right)\right]^{1 / 2}}{2 i k(c \bar{c})^{1 / 2}} .
$$

Now squaring and solving for $k^{2}$, we get the value stated in the theorem.

New Mexico College of Agriculture and Mechanic Arts

* L. R. Ford, Automorphic Functions, p. 28. 